\title{
Correspondence
}

We welcome letters to the Editor concerning articles which have recently been published. Such letters will be subject to the usual stages of selection and editing; where appropriate the authors of the original article will be offered the opportunity to reply.

Letters should normally be under 300 words in length, double-spaced throughout, signed by all authors and fully referenced. The edited version will be returned for approval before publication.

\section{Management of chronic pain in whiplash injury}

Sir,

We read with interest the study by Alpar et $\mathrm{al}^{1}$ in which they treated 38 whiplash patients (by definition Grade 2 whiplash-associated disorder) with carpal tunnel decompression. As a result, they advocate this surgery in whiplash patients. We believe that it is difficult to accept their conclusion at face value and we would like to raise the following questions:

1. We do not know how Alpar et al's patients were selected. If self-selected it is likely that they would have shown a high rate of recovery regardless of the intervention, or would have been more compliant with, for example, rehabilitation after surgery. This may, in turn, treat a problem which does not relate to recovery from surgery, but nevertheless improves outcome. The same concerns could also be applied to the 30 people who were not reported upon. A randomisation procedure reduces the chance that differences would occur as equal numbers in each group could then refuse surgery.

2. We are also concerned regarding the period of recruitment. Could the experimental group be a group of highly selected subjects, and therefore not similar to other whiplash patients seen in primary care?

3. What were the levels of neuropeptides in the control group? Since the control group seemed to have chronic pain even two years later, what became of the neuropeptide levels?

4. If the majority of subjects had motor weakness, why do they also have normal nerve conduction studies (NCS) and electromyography (EMG)? Contrary to what Alpar et al state, these studies are so sensitive that they detect minor conduction abnormalities or motor dysfunction (even in asymptomatic subjects). ${ }^{2-4}$ The authors may be underestimating the diagnostic capabilities because they reference studies evaluating NCS and EMG carried out over 20 to 30 years ago.

5. The benefits of sham-surgery have been known for many years. When we study a group of whiplash patients, whose symptoms are subjective, without objective measurements of nerve function, should we accept a non-randomised, non-placebo study as the gold standard? When the authors in this study use a local anaesthetic around the median nerve to test for temporary relief

(C)2003 British Editorial Society of Bone and Joint Surgery doi:10.1302/0301-620X 85B6.14648 \$2.00

J Bone Joint Surg [Br] 2003;85-B:931-4. of symptoms, they could have carried out a placebo control study at the same time.

Researchers often find what they are seeking in their studies, and the whiplash industry is one with huge tertiary gains. ${ }^{5}$ Now we have carpal tunnel surgery for non-carpal tunnel syndrome, to treat the neck and shoulder pain of whiplash patients.

If Alpar et al wish to advocate something, it should not be surgery but correct evaluation of this form of therapy.

O. KWAN, PhD (Psych)

J. FRIEL, PhD (Psych)

Private Practice,

Edmonton, Alberta,

Canada.

1. Alpar EK, Onuoha G, Killampalli VV, Waters R. Management of chronic pain in whiplash injury. J Bone Joint Surg [Br] 2002;84-B:80711.

2. Werner RA, Franzblau A, Albers JW, Buchele H, Armstrong TJ. Use of screening nerve conduction studies for predicting future carpal tunnel syndrome. Occup Environ Med 1997;54:96-100.

3. Glass I, Ring H. Median nerve conduction tests and Phalen's sign in carpal tunnel syndrome. Electromyography Clin Neurophysiol 1995;53:107-12.

4. Jitpraphai C, Prachathomrong P, Chira-Adisai W. Subclinical carpal tunnel syndrome in hospital staff. $J$ Med Assoc Thailand 1994;77:517-9.

5. Kwan O, Ferrari R, Friel J. Tertiary gains and disability syndromes. Med Hypo 2001;57:459-64.

Authors' reply:

Sir,

We thank Drs Kwan and Friel for their interest in our paper. In answer to their comments:

1. Carpal tunnel decompression was carried out after whiplash injuries in chronic cases, where pain severely curtailed daily activities. Our patients did not have any rehabilitation treatment after surgery, therefore it had no effect on the recovery.

2. We appreciate the difficulties that our colleagues experience in evaluating the symptoms of chronic whiplash patients. Our cases were referred from primary care and they have the same clinical symptoms as any chronic whiplash injury.

3. The neuropeptide levels in untreated chronic whiplash cases continued to remain elevated, since they are the main pain neurotransmitters.

4. One of the most important findings of our study was that neurophysiological studies were normal. ${ }^{1}$ The same conclusions were reached by Ide et al. ${ }^{2}$ In whiplash cases, pain fibres are mainly affected and routine neurophysiological studies can only detect when myelinated fibres are considerably involved in the pathological process.

Nerve conduction studies, which were regarded until recently, as the gold standard for the diagnosis of typical carpal tunnel syndrome, are now regarded as superfluous for both the diagnosis and the assessment of outcome. Many have queried their value as a diagnostic tool, finding no association between them and a patient's clinical status or satisfaction after surgery. ${ }^{3-6}$ Therefore, we believe that they should be excluded from the diagnostic investigations of atypical carpal tunnel syndrome.

While we accept that tertiary gain is a motive when treating patients with whiplash injury, we believe as indicated in the paper that some patients with chronic arm pain after a whiplash injury 
have an 'atypical' carpal tunnel syndrome and are helped by appropriate surgery.

E. K. ALPAR, MCh Orth, FRCS Ed Orth

G. ONUOHA, $\mathrm{PhD}$

V. V. KILLAMPALLI, DNB Orth, MSc Traumatology

R. WATERS, FRCS (Plast)

University Hospital Seely Oak NHS Trust

Birmingham, UK.

1. Alpar EK. Whiplash injury and median nerve entrapment. Injury 1997;28:243

2. Ide M, Ide J, Yamaga M, Takagi K. Symptoms and signs of irritation of the brachial plexus in whiplash injuries. J Bone Joint Surg $[\mathrm{Br}]$ 2001;83-B:226-9.

3. Braun RM, Jackson WJ. Electrical studies as a prognostic factor in the surgical treatment of carpal tunnel syndrome. J Hand Surgery 1994;19A:893-900.

4. Concannon MJ, Gainor B, Petroski GP, Puckett CI. The predictive value of electrodiagnostic studies in carpal tunnel syndrome. Plast Recon Surg 1997;100:1452-8.

5. Finsen V, Russwurm H. Neurophysiology not required before surgery for typical carpal tunnel syndrome. J Hand Surgery 2001;26B:61-4.

6. Redmond MD, Rivner MH. False positive electrodiagnostic tests in carpal tunnel syndrome. Muscle Nerve 1988;11:511-8.

\section{Extramedullary or intramedullary tibial alignment guides: a randomised, prospective trial of radiological alignment}

Sir,

I read with interest the article in the August 2002 issue by Reed et $\mathrm{al}^{1}$ entitled 'Extramedullary or intramedullary tibial alignment guides: a randomised, prospective trial of radiological alignment'. I personally use both alignment systems combined with navigation and would like to address the following points to the authors.

First, I wonder how they were able to obtain an exact zero degree slope cut with an extramedullary alignment system, since as they suggested, posterior slope and malrotation will influence the tibial position. ${ }^{1-2}$ Often, surgeons prefer the tibial component in slight external rotation, resulting in varus malalignment. ${ }^{2}$

Secondly, what was the standard tibial alignment technique used by each surgeon before this study? If they were predominantly IM guide users, this study reflects rather their own learning curve for the other technique than an objective analysis of both techniques. Furthermore, I noticed that some of the procedures $(16 \%)$ were carried out by trainees. Extramedullary alignment probably requires more surgical experience than IM guides. Are the higher degrees of malalignment $\left(>3^{\circ}\right)$ on the right and left side of the Gauss curve in Figure 2 not suggestive of a learning curve?

Finally, what was the preoperative alignment of the knees in the study group, because IM alignment is more accurate in the varus than the valgus knee due to the bowing of the tibia. ${ }^{3}$ The main advantage of extramedullary alignment is a good result even with the difficult and bowed tibiae, where we could expect some malalignment with an IM guide. ${ }^{4-5}$

\section{E. THIENPONT, MD}

University Hospital St Luc

Brussels, Belgium.

1. Reed MR, Bliss W, Sher JL, et al. Extramedullary or intramedullary tibial alignment guides: a randomised prospective trial of radiological alignment. J Bone Joint Surg [Br] 2002;84-B:858-60.

2. Thienpont E, Bellemans J, Fabry G. Posterior slope cut related to rotational alignment in total knee arthoplasty. J Bone Joint Surg $[\mathrm{Br}]$ 2001;83-B (Suppl. II): 170.

3. Simmons ED Jr, Sullivan JA, Rackemann S, Scott RD. The accuracy of tibial intramedullary alignment devices in total knee arthroplasty. J Arthroplasty 1991;6:45-50.
4. Dennis DA, Channer M, Susman MH, Stringer EA. Intramedullary versus extramedullary alignment systems in total knee arthroplasty. J Arthroplasty 1993;8:43-7.

5. Maestro A, Harwin S, Sandoval MG, Vaquero DH, Murcia A. Influence of intramedullary versus extramedullary alignment guides on fina total knee arthroplasty component position: a radiographic analysis. J Arthroplasty 1998;13:552-8.

\section{Authors' reply:}

Sir,

We thank Dr Thienpont for his interest in our paper. We note the use of intramedullary and extramedullary alignment combined with navigation in his unit.

We recognise that an exact zero slope cannot be guaranteed with extramedullary alignment systems. As explained in the paper, this was selected to minimise the effects of any malrotation on varus/valgus alignment. It might be interesting to compare this plane of alignment (tibial slope) in a future study of cohort patients. Tibial components were placed in neutral rotation and the preoperative alignment was not assessed.

Before the study, three of the four consultants used extramedullary alignment routinely. Since the study, all use intramedullary alignment except in cases unsuitable for this technique (previous fracture, excessive tibial bowing etc). As stated, these were specifically excluded from the trial. We have reanalysed the data including only operations performed by consultants who routinely used the extramedullary technique before the trial began. In this subset, intramedullary alignment is still the superior technique $(\mathrm{p}<$ 0.001 ). This implies, that if a learning curve exists, it must be very long indeed.

M. REED, MD, FRCS (Trauma \& Orth)

P. PARTINGTON, FRCS (Orth)

Wansbeck General Hospital

Ashington, UK.

\section{Complications of arthroscopy of the knee}

Sir,

Even as a hand and upper limb surgeon, I read with interest the article in the September 2002 issue by Allum' ${ }^{1}$ entitled 'Complications of arthroscopy of the knee'. In particular, I was interested to note the high incidence of complex regional pain syndrome. I wonder if I might be allowed a moment of pedantry to point out that while he mentions that formation of a "neuroma can be troublesome and it is possible that a relatively minor nerve injury can lead to a complex regional pain syndrome type 1 ", this is not strictly correct; my understanding of current terminology is that this is, by definition, a complex regional pain syndrome type 2 .

\section{MUIR, MB, MCh (Orth), FRCS (Orth) \\ Hope Hospital}

Salford, UK.

1. Allum R. Complications of arthroscopy of the knee. J Bone Joint Surg [Br] 2002;84-B:937-45.

\section{Author's reply:}

\section{Sir,}

I thank Mr Muir for his interest in my article.

My understanding is that chronic regional pain syndrome type I embraces the post-traumatic reflex sympathetic dystrophy syndrome, which is of uncertain cause. Injury to a nerve may contribute to the symptoms, although if a finite anatomical lesion is present, such as a neuroma, then this, indeed, is chronic regional 
pain syndrome type II, which is really a new definition for what has previously been called causalgia. I apologise for any confusion that may have arisen and thank Mr Muir for pointing out the precise definition.

R. ALLUM, FRCS

Wexham Park Hospital

Slough, UK

\section{Deterioration of long-term clinical results after the Elmslie-Trillat procedure for dislocation of the patella}

Sir,

I read with interest the article in the August 2002 issue by Nakagawa et $\mathrm{al}^{1}$ entitled 'Deterioration of long-term clinical results after the Elmslie-Trillat procedure for dislocation of the patella'.

The authors are to be congratulated on their long-term, followup study. Approximately 50\% of their patients developed patellofemoral pain 10 to 20 years after their procedure. This is, of course, disturbing, but their study group had a Q angle of about $20^{\circ}$ which would qualify as mildly elevated.

One conclusion not discussed by the authors might be to avoid the Elmslie-Trillat operation in patients whose Q angle is only mildly elevated which to some might appear intuitive, since in this setting the tibial tuberosity is to begin with almost normally positioned. It would eventually be of interest to see if patients with greatly increased $\mathrm{Q}$ angles (and a seriously malpositioned tibial tuberosity) also develop patellofemoral pain after the Elmslie-Trillat operation.

R. GRELSAMER, MD

The Corinthian Hospital for Joint Diseases New York, USA.

1. Nakagawa K, Wada Y, Minamide M, Tsuchiya A, Moriva H. Deterioration of long-term clinical results after the Elmslie-Trillat procedure for dislocation of the patella. J Bone Joint Surg [Br] 2002;84-B:861-4.

\section{Author's reply:}

Sir,

I thank Dr Grelsamer for his interest in our article.

We did not show the detailed data in the paper because one of the reviewers did not want to emphasise the results concerning the $\mathrm{Q}$ angles which includes subjective aspects. We mentioned that there was no significant difference in the mean preoperative Q angles between the excellent or good result group and the fair or poor result group $\left(20.3^{\circ}\right.$ and $21.0^{\circ}$ respectively). The table shows the relationship between the clinical results and the $\mathrm{Q}$ angles. There was no significant difference between them (Mann-Whitney test).

\begin{tabular}{lll}
\hline $\begin{array}{l}\text { Preoperative } \\
\text { Q angle }\end{array}$ & No. of cases & $\begin{array}{l}\text { Fulkerson functional } \\
\text { score (Pain score) }\end{array}$ \\
\hline$<15^{\circ}$ & 11 & $88.2 / 100(38.5 / 45)$ \\
$16^{\circ}$ to $25^{\circ}$ & 23 & $85.6 / 100(37.4 / 45)$ \\
$26^{\circ}$ to $31^{\circ}$ & 11 & $84.5 / 100(37.0 / 45)$ \\
$>31^{\circ}$ & 4 & $88.8 / 100(37.0 / 45)\}$ \\
\hline
\end{tabular}

In conclusion, we could not say that the patients with greatly increased preoperative Q angles did not develop patellofemoral pain. We agree that the pressure within the patellofemoral joint of these patients may not increase after operation (medial transfer of the tibial tubercle). However, it is difficult to find an obvious effect of the $\mathrm{Q}$ angle on patellofemoral pain, as many other factors affect the outcome.

K. NAKAGAWA, MD

Rush Medical College

Chicago, Illinois, USA.

\section{Management of completely displaced metaphyseal fractures of the distal radius in children}

Sir,

We read with interest the article in the April 2002 issue by McLaughlan et $\mathrm{al}^{1}$ entitled 'Management of completely displaced metaphyseal fractures of the distal radius in children'. The general message of the paper advocates percutaneous Kirschner (K-) wiring of these sometimes difficult fractures. The authors' protocol "required that they adhered to current hospital procedures", which meant that to remove the K-wires, all 35 patients were subjected to a second hospital admission and a second anaesthetic at three weeks. Their paper could be construed as providing a strong argument against K-wire pinning of these fractures, thus avoiding the need for a second admission and second anaesthetic in $79 \%$ of patients in this group ( 7 of 33 patients, $21 \%$, required remanipulation under anaesthesia).

Although the authors comment that "in practice, many children can tolerate removal of the wire in the outpatient department", they do not strongly advocate such practice and did not follow such a practice for the purposes of their study. They concluded that "the need for follow-up radiographs and further procedures" was reduced in the group managed with supplementary K-wire fixation, a conclusion that we would strongly challenge in the light of their $100 \%$ second admission and anaesthetic rate for these patients.

We would be most interested to hear their views on this matter in the light of their results, which suggest no significant difference in the clinical outcome between the two methods of treatment measured three months after injury. For example, was the need for removal of the wire under anaesthesia deemed a prerequisite by the local Ethical Committee and if so, why?

C. CARPENTER, MB, BCh, MRCS

P. WILLIAMS, MB, MCh, FRCS (Trauma \& Orth)

Swansea NHS Trust

Swansea, UK.

1. McLaughlan GJ, Cowan B, Annan IH, Robb JE. Management of completely displaced metaphyseal fractures of the distal radius in children. J Bone Joint Surg [Br] 2002;84-B:413-7.

\section{Authors' reply:}

Sir,

We thank Ms Carpenter and Mr Williams for their interest in our paper.

The protocol of the study which was submitted to the local Ethics Committee had to adhere to policies within the hospital at the time of the inception of the study, when almost all metalwork was removed under general anaesthesia. The paper went on to extrapolate our results from a tightly controlled protocol to a wider clinical setting, where the majority of children could have their wires removed as an out-patient some three weeks after their operation. We have recently audited our current practice of K-wire removal and over $80 \%$ of children in our hospital have the wires removed without general anaesthesia. 
The focus of the paper was that manipulation under anaesthesia (MUA) plus a single K-wire was significantly better at maintaining an anatomical reduction of a completely displaced distal radial metaphyseal fracture than MUA alone. We believe that this was shown unequivocally. We consider that there is an advantage in using K-wires of this type of fracture, as currently, the majority of wires can be removed without anaesthesia, the K-wire group needed fewer X-rays postoperatively and there was a significant displacement rate in those fractures treated without wires.

G. J. McLAUGHLAN, FRCS (Trauma \& Orth)

I. H. ANNAN, FRCS Ed (Orth)

J. E. ROBB, FRCS Ed

Royal Hospital for Sick Children

Edinburgh, UK.

\section{Fellowship Exam of European Board of Orthopaedics and Traumatology}

The third Fellowship exam of the European Board of Orthopaedics and Traumatology is going to take place in the Onze Lieve Vrouwe Gasthuis in Amsterdam, Holland on the

\section{1th and 12th October, 2003.}

\section{Requirements}

1. Citizenship of European Union (EU) including Norway and Switzerland, and New Countries joining the European Union (EU).

2. Certificate of full qualification as an Orthopaedic Surgeon by the National Authorities

(EU Orthopaedic Society).

3. Be fluent in English.

Title

"Fellow of the European Board of Orthopaedics and Traumatology"

Enquiries

ebotexam@mail.telepac.pt

www.ebotexam.20m.com

*Please read the information that will be published in the European Orthopaedic literature or contact your national UEMS-European Board of Orthopaedics and Traumatology delegate.

\section{Application}

Deadline for application will be the 31st of July 2003.

\section{Number of candidates restricted to $\mathbf{4 0}$}

